\title{
The study of locomotor pointing in virtual reality: The validation of a test set-up
}

\author{
A. DE RUGY and G. MONTAGNE \\ CNRS and Université de la Méditerranée, Marseille, France \\ M. J. BUEKERS \\ Katholieke Universiteit, Leuven, Belgium \\ and \\ M. LAURENT \\ CNRS and Université de la Méditerranée, Marseille, France
}

\begin{abstract}
The goal of this experiment was to validate an experimental set-up for studying locomotor pointing. The specific and also original element of this set-up was the interactive nature of virtual reality and movement production. This interaction was achieved through the coupling of a treadmill and a Silicon Graphics system. This latter system generated on a screen $(3 \times 2.3 \mathrm{~m})$ an environmental array that moved according to the action produced by subjects on a treadmill. The task was to place either foot on a spatial target that appeared on the floor in front of the subject's displacement trajectory. We analyzed the step length patterns of subjects approaching these targets, along with the current target-subject relationship. The results are in agreement with a perception-action coupling type of control mechanism that operates continuously as the subject approaches the desired target. Apparently, these findings mirror observations of real-life locomotion, indicating that the present set-up provides a valid and useful tool for examining human locomotion.
\end{abstract}

Locomotor pointing, in which either foot is placed in a precise location while maintaining locomotion, is a widely used paradigm in visuolocomotor coordination studies. Recently, some of those studies have made use of virtual reality to examine these basic movement patterns. However, these studies were limited to the presentation of specific optical flow patterns by means of a computer, without taking into account the important interaction between perception and action. That is to say, the actions produced by subjects have a direct effect on the optic flow pattern. In the present experiment, we present an original test set-up and method that enables this perception-action interaction. Moreover, we want to examine whether this set-up provides a valid tool for studying human locomotion. This validation can be obtained by examining whether the control processes underlying locomotion in a real-life setting and a virtual reality setting are similar. Thus, before going into the specifics of the apparatus, we will first focus on how locomotor pointing is controlled.

Lee, Lishman, and Thomson (1982) initially showed that the intertrial variability of the toe-board distance (in long jumping) for the subsequent support phases of the approach decreased during the last few strides preceding

Correspondence concerning this article should be addressed to: A. de Rugy, Université de la Méditerranée, Faculté des Sciences du Sport, UMR Mouvement et Perception, 163 Avenue de Luminy CP 910 , 13288 Marseille Cedex 9, France (e-mail: de_rugy@ laps.univ-mrs.fr). the jump. Several studies based on the same intertrial variability analysis provided support for these findings and hence confirmed the use of visual control during the last few strides of the approach (Berg, Wade \& Greer, 1994; Hay, 1988; Scott, Li \& Davids, 1997). Hay showed that this control generally began five strides from the board, despite the individual differences that were observed between subjects. Berg et al. and Scott et al. reinforced this idea that stride number was the intrinsic unit used by subjects (in their case four strides from the board), rather than absolute distance. However, the presumed stability of this stride number poses some problems regarding the control mechanism used.

The idea of stride number stability is certainly compatible with an approach that incorporates a feedforward type of control (e.g., Jordan, 1990), which is claimed to intervene at a precise moment in the approach. However, it is opposed to the perception-action coupling concept $(\mathrm{Ku}-$ gler \& Turvey, 1987), which is built on the existence of a circular causal relationship between perception and action. According to this concept, the action is a function of the current situation, available through the subject's perception of the optical flow generated by his/her displacements in the environment. Since the amount of adjustment required for arriving at the target varies throughout the successive trials, the initiation of the regulation at a stable stride number does not seem very useful. Actually, this regulation strategy has been challenged by Montagne, Cornus, Glize, Quaine, and Laurent (2000). Using a trial-by-trial analy- 


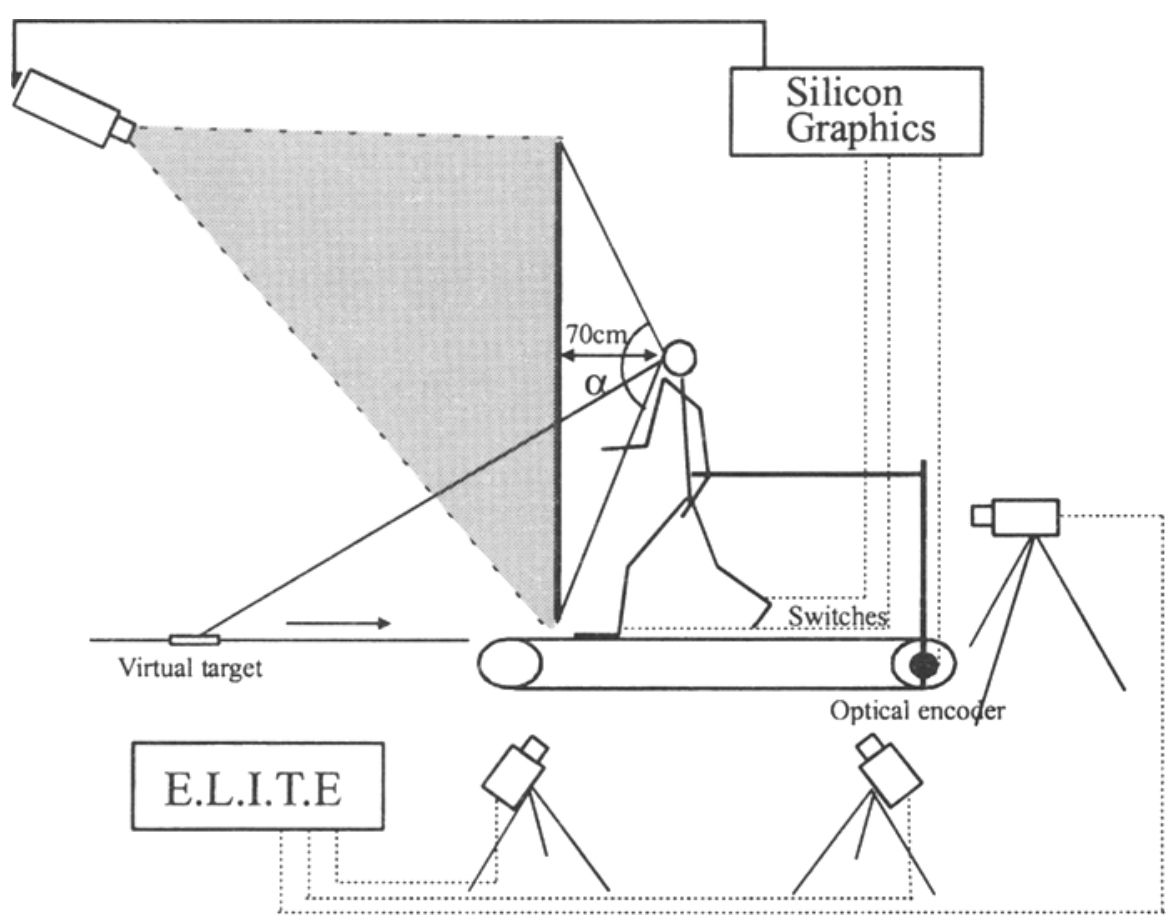

Figure 1. Diagram of the apparatus. The subject walks on the treadmill while controlling the velocity of the moving belt. This velocity, in turn, produces a proportional movement of the virtual optical array. The subject, who is immersed in an interactive virtual environment, regulates his/her step length in order to be able to accurately point at the approaching target.

sis, these authors examined the actions of the subjects according to the characteristics of the current situation. This procedure made it possible to relate the adjustments made by the subjects to the adjustments that were required, as well as to determine the step number at which these regulations were initiated. The results indicated that the control mechanism involved is effectively based on a continuous perception-action coupling.

An interesting approach for investigating these control mechanisms is to manipulate the optical flow available to the subjects. As we stated above, we will use an apparatus allowing us to explicitly define the optical flow as a function of the displacements of the subjects. In this study, we want to examine whether a similar control mechanism (namely, one of perception-action coupling) is found for subjects executing a locomotor pointing task in a virtual reality setting. If so, the apparatus would be a valid tool with which to further investigate human locomotion pointing.

\section{METHOD}

\section{Subjects}

Data for 2 female subjects ( 22 and 24 years of age) are reported in this experiment.

\section{Apparatus}

The purpose of this apparatus was to record the characteristics of a subject's locomotion while walking on a treadmill and perform- ing a locomotor pointing task in a virtual environment. The control of the optical flow was attained through the use of a Silicon Graphics animation system that immersed the subject in a virtual environment. The treadmill was coupled to the Silicon Graphics system so that the speed of the movement produced by the subject generated the appropriate environmental flow on the screen. Kinematic data associated with the subject's locomotion was recorded by using the ELITE movement acquisition system.

Treadmill. The subjects walked along the treadmill's (Gymrol, Model BRL 1800$)$ moving belt $(0.6 \mathrm{~m}$ wide $\times 1.8 \mathrm{~m}$ long), which glided over an entirely flat and rigid surface. The subject was attached to the framework of the treadmill by means of a weight lifting belt that was fixed to a rotating axis via a rigid rod (see Figure 1). This device enabled the forces that were generated by the subject while walking to be transmitted to the treadmill. An internal control system subsequently allowed the initial velocity of the belt to be modulated, in order to help the subject achieve a regular walking pace. This "aid" was chosen so that the forces generated by the subject would result in a velocity of the moving belt that was practically equivalent to the velocity that would have resulted if the same forces were generated by the subject while walking on a normal surface. Thus, the subjects regulated their locomotion through the modulations in the traction force on the weight lifting belt. The treadmill also contained an optical encoder that measured, at a rate of 1 pulse $/ \mathrm{mm}$, the distance covered by the subject.

Footswitches. Footswitches were attached to the heels of the subjects' feet in order to give the temporal positioning of the feet. The signals that originated from both switches and from the optical encoder were fed into the Silicon Graphics system (see Figure 1). From the information provided by the footswitches and the optical device that measured the distance covered by the subject, the image of the virtual environment was calculated. 
Computation of the images. The successive images, which represented the actions of the subject, were computed and projected as follows. At any given time $t 1$, Image 2 was computed from the parameters that were available to the computer during the time that Image 1 was projected on the screen. For the Silicon Graphics Indy system, the calculation time for one image is $42 \mathrm{msec}$. During this time, the system integrated the data from the treadmill and the footswitches. Thus, at a time $t 1+42 \mathrm{msec}(t 2)$, Image 2 was computed and appeared on the screen. At this time, the computation of the following image (Image 3 ) began. Thus, when the subject accelerated at a given time $t$, situated between $t 1$ and $t 2$, the results of this acceleration were integrated by the computer at time $t 2$. The image that results from this acceleration was calculated between $t 2$ and $t 3$ and projected onto the screen at time $t 3$. The visual information of the acceleration was therefore only available after a delay of between 42 and $84 \mathrm{msec}$.

The visual scene. The visual scene-namely, a three-dimensional hallway-was computed from the condition of the switches, the data from the optical encoder of the treadmill, and two additional preset parameters (subject's eye height and distance of the subject's eyes from the screen; see Figure 1). The height and the width of the virtual hallway (that extends to infinity) were 3 and $3.2 \mathrm{~m}$, respectively. The walls were textured with a pattern of random forms and colors. At any given moment, a virtual rectangular target $(0.5 \mathrm{~m}$ long $\times 0.1 \mathrm{~m}$ wide) could be presented at a chosen distance. This target was projected on the floor in the center of the hallway. The overall visual scene was projected onto a screen ( $3 \mathrm{~m}$ wide $\times 2.3 \mathrm{~m}$ tall) positioned in front of the subject, so that the eyes were at about $0.7 \mathrm{~m}$ from the screen. The subjects also wore a special mask that restricted the angles of their visual field to $110^{\circ}$ in both the vertical (see angle $\alpha$ in Figure 1) and the horizontal axes. This ensured that the subjects were immersed in the virtual environment.

The appearance of the target. A stride or a complete locomotor cycle (i.e., two successive steps) was used to define the distance at which the target appeared at any particular randomly defined footfall. The distance at which the target appeared varied randomly between 5.5 and 6 times the length of the current stride (i.e., the stride being executed before the target appeared), which represented a distance of about 11-12 steps. Once the subject crossed the target, a few steps (3-4) were taken before the new target appeared.

The analysis of the kinematics. The kinematic movement parameters were obtained by means of an ELITE system (Ferrigno \& Pedotti, 1985). Three cameras, sampling at $100 \mathrm{~Hz}$, were used to collect the kinematic data. Two of these cameras were positioned $3.5 \mathrm{~m}$ to the left of the subject and parallel to the treadmill. The third camera was placed $1.5 \mathrm{~m}$ behind the treadmill in order to record a marker placed at the subject's hip. By using these cameras, a three-dimensional recording of the movements of each of the markers on the subjects was obtained. Five hemispherical markers $(1 \mathrm{~cm}$ in diameter) that reflected infrared light were used. However, only two of these markers, one attached to the external malleolus of the left leg and one to the internal malleolus of the right leg, were analyzed in this study.

The data acquisition synchronization. The start of the data acquisition on the Silicon system simultaneously triggered the acquisition on the ELITE system. The synchronization of the data from both systems was obtained by using an additional trigger.

Data reduction. The data obtained via the ELITE and the Silicon systems allowed us to examine the relationship between the kinematics of the markers and the virtual environment and, more specifically, the target. The kinematics of foot displacement were used to identify the real positions of the feet at footfalls. These were combined with the virtual position of the target at the corresponding instants to obtain the successive footfalls-target distances. The length of the consecutive steps was deducted from these successive dis. tances. A more precise analysis of these step lengths was obtained that was exclusively based on the kinematics data recorded by the ELITE system. The precision of this analysis was on the order of $0.02 \mathrm{~m}$.

\section{Experimental Conditions}

Each subject was exposed to two different experimental conditions, no-pointing and pointing. In the no-pointing condition, the subjects were asked to walk as naturally as possible and to ignore the targets on the floor of the hallway. In the pointing condition, however, the subjects were asked to place their toes on the center of the target while walking.

\section{Procedure}

After defining the speed at which the subject walked on a regular surface at a natural pace, she was placed on the treadmill. With the subject's verbal consent, the "aid" device was adjusted so that the subject felt as though she were walking at a natural pace. The subject's velocity while walking on the treadmill did not differ from the velocity generated during normal walking by more than $10 \%$. Once these parameters were set, the subject had to walk on the treadmill for $2 \mathrm{~min}$ in order to become familiar with the test situation. After a pause of $2 \mathrm{~min}$, the subject walked on the treadmill for an additional 4 min, during which the visual scene of the hallway was presented. During this time, the subject could experience how her walking speed affected her passage through the hallway. Two experimental sessions of $4 \mathrm{~min}$ each were then presented to the subject. These sessions included the no-pointing condition and were separated by a rest period of $2 \mathrm{~min}$. The subject was then given a 4-min familiarization period with the pointing condition before she began the four experimental sessions. These pointing condition experimental sessions were separated by a rest period of $2 \mathrm{~min}$.

Data analysis. First, the precision of the pointing task was examined. This was followed by the two trial-by-trial analyses proposed by Montagne et al. (2000) - namely, an intrastep number analysis and an interstep number analysis. To examine pointing precision and locomotor adjustments in the no-pointing condition, we considered the footfall that was closest to the target to be the pointing footfall.

Pointing precision. The pointing precision was examined by means of the absolute, constant, and variable error scores. The absolute error (AE) was defined as the absolute mean difference between the position of the toes and the center of the target at the last footfall. For the constant error (CE), the signed mean difference (i.e., the distance between the toes and the target at the last footfall) was used (there was a negative difference when the toes were placed behind the target and a positive difference when the toes were placed in front of the target). The variable error (VE) represents the standard deviation of the distance between the positions of the toes and the target at the last footfall.

Intrastep number analysis. For each trial, we examined the relationship between the adjustment that was made and the adjustment that needed to be made to accurately point the target. The sign of these adjustments was also taken into account (i.e., the shortening or lengthening of the steps). The adjustment made represents the difference between the current step length and the mean step length for that given step number. The required adjustment represents the difference between the current toe-target distance and the mean toe-target distance at the given step number. A description of step length and toe-board distances for given step numbers is shown (see Figure 2). To evaluate the coherence of the adjustments made (i.e., their correspondence with the adjustment needed), the adjustment produced at a given step number $(n)$ was related to the adjustment needed at the previous step number $(n-1)$. A linear regression analysis was used to examine this relationship.

Interstep number analysis. For each trial, the step number at which regulation was initiated and the amount of adjustment produced during this regulation were calculated, in order to evaluate 


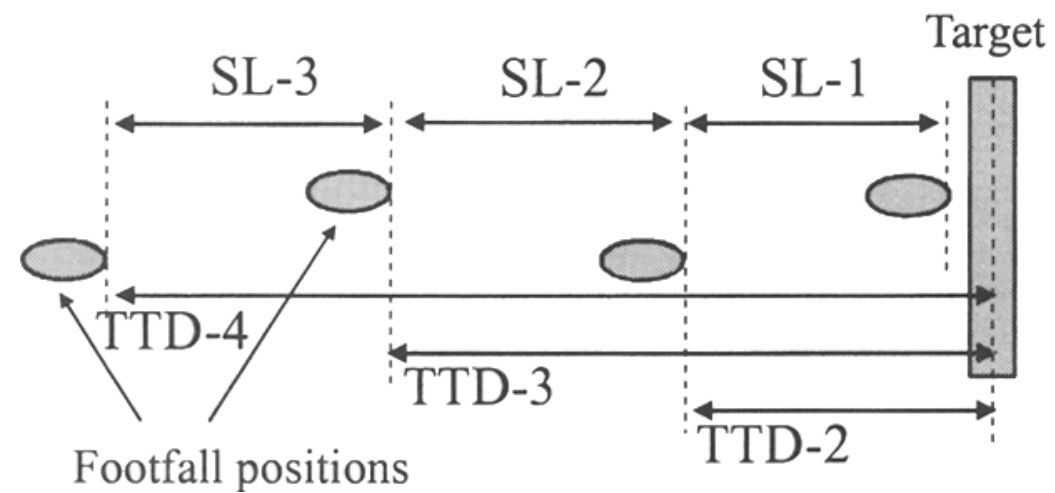

Figure 2. This figure shows how the step numbers correspond to the subject's footfalls. The arrows below indicate the toe-target distances (TTD) for the corresponding step numbers $(-4$ to -2$)$. The upper arrows indicate the step length (SL) for the given step numbers $(-3$ to -1$)$.

the possible link between these two variables. For each trial, the means and standard deviations of four successive steps registered during the steady phase of the displacement - that is, during the initial nonregulated phase of the approach-were calculated. This mean value represented the standard step that was used to compare each of the steps of the given trial. The trial was considered as being regulated from a given step number when the length of the step at that given step number was smaller or larger than the standard step by $6 \mathrm{~cm}$. From this step number, the amount of adjustment was calculated for the nonmixed trials (i.e., trials in which the steps were either all lengthened or all shortened). This adjustment corresponded to the sum of the differences between each adjusted step and the standard step. Finally, a regression analysis was performed between the amount of adjustment and the step number at which regulation was initiated.

In this study, the mixed trials (i.e., trials containing a mixture of steps that were lengthened and shortened) were not taken into account, since their function is not very well understood (see, e.g., Montagne et al., 2000). Moreover, the calculation of the amount of adjustment, as proposed above, poses some methodological problems, since this amount fails to correctly represent the required adjustment. For example, a prolonged step followed by a shortened one can produce the same result as two standard steps. For these reasons, the mixed trials were excluded from the analysis.

\section{RESULTS}

\section{Pointing Precision}

For the pointing condition, the mean error scores were smaller $(\mathrm{AE}=0.15 \mathrm{~m}, \mathrm{CE}=0.02 \mathrm{~m}$, and $\mathrm{VE}=0.18 \mathrm{~m})$ than those for the no-pointing condition $(\mathrm{AE}=0.24 \mathrm{~m}$, $\mathrm{CE}=0.06 \mathrm{~m}$, and $\mathrm{VE}=0.30 \mathrm{~m}$ ).

\section{Intrastep Number Analysis}

The analysis was conducted on 36 no-pointing trials and on 82 pointing trials, from the step number -5 to the step number -1 (i.e., the actual pointing). A significant $(p<.05)$ linear relationship was found between the two variables (the adjustment needed and the adjustment produced) for the pointing condition but was absent in the no-pointing condition (see Figure 3A). This relationship became significant at step number -3 and persisted up to step number -1 (see Figure 3B).

\section{Interstep Number Analysis}

The analysis showed that $56 \%$ of the trials were regulated $(n=46), 65 \%$ of which were nonmixed $(n=30)$. For these latter trials, the step number at which the initiation of regulation occurred was spread out from step number -3 to step number -1 . The linear regression analysis conducted between the amount of adjustment made and the step number at which the regulation was initiated revealed a significant link between these two variables $(p<.05)$. The greater the amount of adjustment, the sooner the subject initiated the regulation $(y=12.7+$ $\left.1.6 ; R^{2}=.61\right)$.

\section{DISCUSSION}

The high level of precision achieved by the subjects in the pointing task condition can be explained solely by the visually guided step length regulation, which was absent in the no-pointing condition. This finding, which has previously been found to exist in natural situations, would appear to provide the first fundamental step toward validating our apparatus.

The search for the control mechanisms underlying these regulations led us to the trial-by-trial analysis, which provides a means of relating the actions of the subject to the requirements of the current situation. The intrastep number analysis asserts the importance and coherence of these regulations, since it associates the adjustments produced during the last steps with the adjustments required - that is, the subject's behavior depends on the current situation, which in turn is specific for a given trial. In addition, the interstep number analysis indicates that subjects initiated their regulations sooner when the amount of adjustments were more substantial. In line with Montagne et al.'s (2000) data, our results support the 

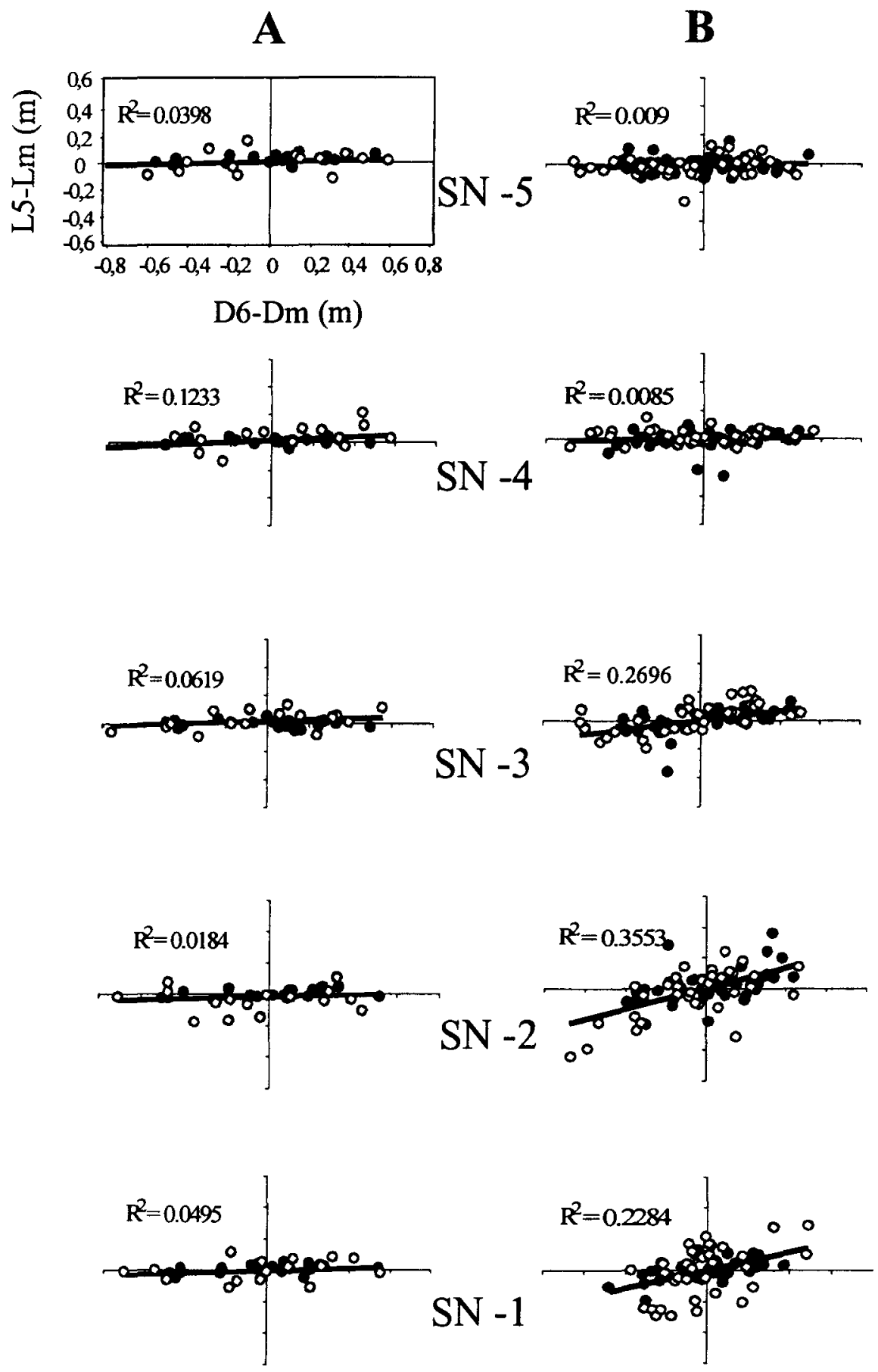

Figure 3. This diagram shows the relationship between the adjustment made (y-axis) and the adjustment required in order to reach the target ( $x$-axis), for each step number (from $\mathrm{SN}-5$ to $\mathrm{SN}-1)$ and for each trial. A represents the trials collected in the no-pointing condition $(n=36)$, and $B$ represents the results obtained in the pointing condition $(n=82)$. The adjustment produced at a step number $n$ is the difference between the current step length ( $\mathrm{Ln}$ ) and the mean step length $(\mathrm{Lm})$ at this step number. The required adjustment at a step number $n$ is the difference between the current toe-target distance $\left(D_{n-1}\right)$ and the mean toe-target distance $(D m)$ for the previous step number. The filled circles represent one subject, whereas the open circles represent the other. Although absent in the no-pointing condition (A), a linear relationship between those two variables $(p<.05)$ appears at $\mathrm{SN}-3$ in the pointing condition $(B)$. This relationship persists until the end of the approach. 
funnel-like type of control that was proposed by Bootsma, Houbiers, Whiting, and Van Wieringen (1991) in order to explain the visual control of goal-directed actions. The spatiotemporal tolerance of the behavior that is indexed to the time to contact (TTC) characterizes this type of control and is actually found in the present locomotor pointing task. Hence, the stability of the stride number at which the regulation is initiated (Berg et al., 1994; Scott et al., 1997), evoking a feedforward planning at this point of the regulation, should be considered as an artifact that is due to limitations of the intertrial analysis. In fact, the trial-by-trial analyses highlight a control mechanism based on a perception-action coupling that continuously operates during the approach.

These findings mirror observations of real-life locomotion, indicating that the present virtual reality test setup provides a valid and useful tool for examining the relationship between perception and action operating during locomotor pointing. Moreover, in controlling the optical flow as a function of the subject's displacements, we will now be able to formally test the hitherto hypothetical use of the optical variable tau (Lee, 1976) while performing locomotor pointing (Berg et al., 1994; Lee et al., 1982). The optical variable tau (i.e., the inverse of the rate of dilation of the retinal image of an object) specifies the TTC between a subject and an object, traveling at a constant relative speed on the same trajectory. The use of tau in ball catching was elegantly demonstrated through an experimental manipulation of ball expansion (Savelsbergh, Whiting, \& Bootsma, 1991). The present virtual reality test set-up will allow us to perform similar manipulations during locomotor pointing - for example, to confront subjects with a situation in which the actual (physical) size of the target changes during the approach. The resulting expansion will be different from the normal optical expansion of a nonchanging target that approaches a subject. On the other hand, Laurent, Paul, and Cavallo (1988) report the influence of visual speed information provided by peripheral vision, using a locomotor pointing task performed on an airport's conveyor belt moving in the walking direction (so that moving speed in the peripheral vision was higher than the actual walking speed). The present virtual reality test set-up permits us to test whether reduced speed information provided by peripheral vision will also have an effect on pointing performance and/or whether a differently structured environment will have distinct effects on pointing performance. Yet, a number of problems should be solved in order to improve the present set-up. For instance, to study the effects of binocular versus monocular viewing on catching (e.g., Olivier, Weeks, Lyons, Ricker, \& Elliott, 1998), some further adaptations would be needed (e.g., to create disparity).

To conclude, we also know that for practical reasons, treadmills are generally used as a rehabilitation tool. The apparatus presented here could be used to reintroduce to patients, in more secure surroundings, the environmental adaptations that are necessary for successful locomotor rehabilitation.

\section{REFERENCES}

BERG, W. P., WADE, M. G., \& GREER, N. L. (1994). Visual regulation of gait in bipedal locomotion: Revisiting Lee, Lishman, and Thomson (1982). Journal of Experimental Psychology: Human Perception \& Performance, 20, 854-863.

Bootsma, R. J., Houbiers, M., Whiting, H. T. A., \& Van Wieringen, P. C. W. (1991). Acquiring an attacking forehand drive: The effects of static and dynamic experimental conditions. Research Quarterly for Exercise \& Sport, 62, 276-284.

Ferrigno, F., \& PEDotTi, A. (1985). EliTE: A digital dedicated hardware system for movement analysis via real-time TV signal processing. IEEE Transactions on Biomedical Engineering 32, 943-950.

HAY, J. G. (1988). Approach strategies in long jump. International Journal of Sport Biomechanics, 4, 114-129.

JoRDan, M. (1990). Motor learning and the degrees of freedom problem. In M. Jeannerod (Ed.), Attention and performance XIII: Motor representation and control (pp. 796-836). Hillsdale, NJ: Erlbaum.

Kugler, P. N., \& TurveY, M. T. (1987). Information, natural law, and self assembly of rhythmic movements. Hillsdale, $\mathrm{NJ}$ : Erlbaum.

Laurent, M., Paul, P., \& Cavallo, V. (1988). How is gait visually regulated when the head is travelling faster than the legs? Journal of Motor Behavior, 20, 301-316.

LEE, D. N. (1976). A theory of visual control of braking based on information about time-to-collision. Perception, 5, 437-459.

Lee, D. N., Lishman, J. R., \& Thomson, J. A. (1982). Regulation of gait in long jumping. Journal of Experimental Psychology: Human Perception \& Performance, $8,448-458$.

Montagne, G., Cornus, S., Glize, D., Quaine, F., \& Laurent, M. (2000). A "perception-action coupling" type of control in longjumping. Journal of Motor Behavior, 32, 37-44.

Olivier, I., Weeks, D. J., Lyons, J., Ricker, K. L., \& Elliott, D. (1998). Monocular vision in one-hand catching: Interocular integration. Journal of Motor Behavior, 30, 343-351.

Savelsbergh, G. J. P., Whiting, H. T. A., \& Bootsma, R. J. (1991) Grasping tau. Journal of Experimental Psychology: Human Perception \& Performance, 17, 315-322.

ScotT, M. A., LI, F. -X., \& Davids, K. (1997). Expertise and the regulation of gait in the long jump approach phase. Journal of Sports $S \mathrm{Ci}$ ences, 15, 597-605.

(Manuscript received November 9, 1999; revision accepted for publication July 21,2000 .) 